Margherita Amadi, Silvia Visentin*, Francesca Tosato, Paola Fogar, Giulia Giacomini, Giulia Res, Luca Bonadies, Patrizia Zaramella, Mario Plebani, Erich Cosmi and Eugenio Baraldi

\title{
Neonatal lymphocyte subpopulations analysis and maternal preterm premature rupture of membranes: a pilot study
}

https://doi.org/10.1515/cclm-2021-0375

Received March 28, 2021; accepted May 17, 2021; published online June 7, 2021

\section{Abstract}

Objectives: Preterm premature rupture of membranes (pPROM) causes preterm delivery, and increases maternal T-cell response against the fetus. Fetal inflammatory response prompts maturation of the newborn's immunocompetent cells, and could be associated with unfavorable neonatal outcome. The aims were (1) to examine the effects of pPROM on the newborn's and mother's immune system and (2) to assess the predictive value of immune system changes in neonatal morbidity.

Methods: Mother-newborn pairs (18 mothers and 23 newborns) who experienced pPROM and controls (11 mothers and 14 newborns), were enrolled. Maternal and neonatal whole blood samples underwent flow cytometry to measure lymphocyte subpopulations.

Results: pPROM-newborns had fewer naïve CD4 T-cells, and more memory CD4 T-cells than control newborns. The effect was the same for increasing pPROM latency times before delivery. Gestational age and birth weight influenced maturation of the newborns' lymphocyte subpopulations and white blood cells, notably cytotoxic T-cells, regulatory T-cells, T-helper cells (absolute count),

*Corresponding author: Silvia Visentin, MD, PhD, Obstetrics and Gynecology Clinic, Department of Women's and Children's Health, University of Padova, 35128 Padova, Italy, Phone: +390498213400 , Fax: +39 0498218085, E-mail: silvia.visentin.1@unipd.it Margherita Amadi, Giulia Res, Luca Bonadies, Patrizia Zaramella and Eugenio Baraldi, Neonatal Intensive Care Unit, Department of Women's and Children's Health, University of Padova, Padova, Italy Francesca Tosato, Paola Fogar and Mario Plebani, Department of Laboratory Medicine, Padova University Hospital, Padova, Italy. https://orcid.org/0000-0002-0270-1711 (M. Plebani)

Giulia Giacomini and Erich Cosmi, Obstetrics and Gynecology Clinic, Department of Women's and Children's Health, University of Padova, Padova, Italy and CD4/CD8 ratio. Among morbidities, fewer naïve CD8 T-cells were found in bronchopulmonary dysplasia (BPD) ( $p=0.0009)$, and more T-helper cells in early onset sepsis $(\mathrm{p}=0.04)$.

Conclusions: pPROM prompts maturation of the newborn's T-cell immune system secondary to antigenic stimulation, which correlates with pPROM latency. Maternal immunity to inflammatory conditions is associated with a decrease in non-major histocompatibility complex (MHC)restricted cytotoxic cells.

Keywords: immunity; neonatal care; pPROM; preterm delivery; T-lymphocyte.

\section{Introduction}

The most common causes of preterm birth are uncontrollable preterm labor, preterm premature rupture of membranes (pPROM), and iatrogenic maternal-fetal obstetric complications.

Worldwide, pPROM complicates up to $3 \%$ of pregnancies, being responsible for approximately one in three of all preterm deliveries [1]. The latency between the rupture of membranes (23rd and the $36^{6 / 7}$ th week of gestation) and the onset of labor ranges from 0 to 59 days [2]. One of the main causes of pPROM is chorioamnionitis, or intra-amniotic infection [3]. The combination of pPROM and chorioamnionitis is associated with an increased neonatal mortality and morbidity, even after accounting for the degree of prematurity [3]. Some of the most severe consequences of prematurity include cerebral palsy, mental disability, and bronchopulmonary dysplasia (BPD) [4].

Generally speaking, preterm newborns' immune function is immature at birth. Their immunity may be further impaired by factors associated with maternal therapies, such as the use of antenatal glucocorticoids. Type of delivery and admission to the neonatal intensive care unit (NICU) are other variables that affect the immune system and the risk of neonatal infections. 
The neonatal adaptive immune system is known to lack specificity and memory. It continues to mature during the first years of life [5], during which time the newborns' innate immunity represents their first defense against various pathogens. There is a gradual drop in the number and function of some cells, such as monocytes and NK cells, and in the expression of MCH molecules. Meanwhile, pattern recognition receptor activity also tends to decline, especially up to the 34th week of gestation, ultimately increasing early preterm newborns' risk of infection [6]. Preterm newborns also have low total lymphocyte counts, mainly consisting of naïve cells. Their postnatal activation is triggered by foreign antigens, which stimulate an immunological memory. To maintain fetal-maternal immune tolerance, T-helper 2 cells and regulatory T-cells are much more abundant in preterm than at-term newborns [4].

Neonatal antibody production is limited during the first month of life, and lacking in the normal CD40L expression on the surface of T-lymphocytes. In this condition, maternal IgG in the placenta and IgA in breastmilk ensure their passive immunity [4].

On the other hand, the intrauterine environment influences the maternal-fetal immune balance in the event of acute or chronic inflammation. An intrauterine infection, often secondary to-pPROM, can lead to fetal inflammatory response syndrome (FIRS), which is characterized by an increasing inflammatory cytokine secretion and rising levels of T-helper 1 cells [4].

pPROM seems to be associated with an increased maternal T-cell response against the fetus in semiallogeneic form, corresponding to a reduced immune tolerance during pregnancy [7]. T-cells are key players in immune regulation: while regulatory $\mathrm{T}$-cells have a role in suppressing inflammatory response, T-helper 17 cells (induced from naïve T-cells in peripheral blood by antigen priming) are involved in immune response to extracellular pathogens and tissue-specific auto-immune disease.

The aims of the present study were: (1) to examine the effects of pPROM on the newborn's and mother's immune system and (2) to assess the predictive value of immune system changes in neonatal morbidity.

\section{Materials and methods}

\section{Experimental design}

This study was part of a broader investigation on the prevention of preterm birth (Prot. n. 2980P). Signed informed consent forms were obtained from all participating parents. This was an observational study conducted at the high-risk unit of Padua University's Obstetrics and Gynecology Clinic and NICU from November 2018 to September 2019.

Women experiencing PPROM were enrolled at the time of their hospitalization, and their maternal and obstetric data were recorded. Preterm newborns were enrolled on admission to the NICU after delivery. The group of cases included 23 preterm newborns (of whom two twins and one triplet) (median gestational age [GA] 30 $4 / 7$ weeks and birth weight [BW] 1,426 g), and their 18 mothers (median age 34 years), whose pregnancies were complicated by pPROM. Our inclusion criteria were: a diagnosis of pPROM or the onset of uncontrollable preterm labor (at $23-36^{6 / 7}$ weeks of gestation), both associated with a histologically-placenta report. pPROM was defined as the vaginal loss of amniotic fluid and possible presence of oligohydramnios. Colorimetric tests were used to assess $\mathrm{pH}$ or protein levels in the amniotic fluid. Clinical signs of chorioamnionitis (histologically-confirmed chorioamnionitis [hCA]) included maternal temperature, leukocytosis, and smelly amniotic fluid.

Our control group included 14 preterm newborns (median GA 31 weeks and BW 1,450 g) and their 11 mothers (median age 34 years), whose pregnancies were not complicated by pPROM. The control group included preterm mother-newborn pairs hospitalized for maternal or fetal pathological conditions other than clinical or biochemical signs of infectious disease. Data were obtained from women who presented with pre-eclampsia $(\mathrm{n}=1)$, emolysis, elevated liver enzymes, low platelet count (HELLP) syndrome $(n=2)$, maternal tachycardia $(n=2)$, intrauterine growth restriction $(n=3)$, or uncontrollable preterm labor without hCA $(n=3)$.

The groups of cases and controls were comparable in terms of GA at enrollment.

Blood samples were routinely collected in newborns and their mothers, from the umbilical catheter and a peripheral vein, respectively, within 3 days after delivery, for laboratory tests, including immunophenotyping and biochemical markers. All maternal and neonatal outcomes were recorded, as well as all placental histological findings to confirm or exclude the suspicion of chorioamnionitis. Histologically, chorioamnionitis (the presence of more than 10 polymorph nuclear leukocytes per high-powered field) [8] is defined as an inflammatory process affecting the chorion and amnion (chorioamnionitis) or villous tree (villitis), or extending to the umbilical vessels or Wharton's jelly (funisitis), which is a part of FIRS [9].

\section{Patient care}

Maternal antibiotic treatment was begun on admission to hospital, based on our internal protocol. Blood tests were performed repeatedly to check for any presence of inflammatory or infectious disease. Ultrasound and cardiotocography were used repeatedly to monitor fetal well-being. Vaginal swabs and urine cultures were obtained to check for subclinical infection.

Antibiotics were administered prophylactically to all newborns in the NICU, and stopped when sepsis-screening results were negative (based on total WBC counts, absolute neutrophil counts, C-reactive protein [CRP] levels, and bacterial cultures). Early onset sepsis is defined as the onset of lab/symptoms before 3 days of age [10]. As our standard non-invasive mechanical ventilation system (nasalcontinuous positive airways pressure [N-CPAP] and nasal-intermittent mechanical ventilation [NIMV]), we used the Hudson prongs (Hudson 
Respiratory Care, Temecula, CA, USA) or a Vygon cannula (VYGON Italia srl, Gruppo Vygon, Italy, E-mail: ti.nogyv@ofni) connected to a Babylog VN500 (Dräger Medical AG \& Co. KGaA, Lübeck Germany), or a heated and humidified high-flow nasal cannula (HHNC) (http:// www.neotechproducts.com/product-catalog/neotech-ram-cannula). The criteria for administering N-CPAP/NIMV treatment were clinical signs of increased breathing effort (i.e., increased respiratory rate, retractions of the lower ribs and sternum, grunting) with a preserved respiratory function, and a FiO2 demand no higher than 0.30. Most patients with an increased oxygen demand may have needed one or two doses of surfactant and synchronized intermittent mandatory ventilation. BPD is the respiratory disease of preterm newborns, defined as an oxygen requirement $>21 \%$ at 36 weeks postmenstrual age.

\section{Laboratory tests: immunophenotyping and biochemical markers}

All blood samples were obtained in EDTA-anticoagulated tubes, and analyzed on the same day, within 3 days after delivery, to avoid any decrease in cell levels. Samples were prepared in accordance with the CLSI (H42-A2) guideline. In particular, 50-100 mL aliquots of blood were incubated for $15 \mathrm{~min}$ at room temperature with combinations of optimal aliquots of the following fluorochrome-conjugated monoclonal antibodies: CD45-FITC, CD3-PECY5, CD4-PE, CD8-ECD, CD56/16-PE, CD19-ECD (combined in Cyto-Stat Tetrachrome BeckmanCoulter), CD45RA-FITC (Becton-Dickinson), CD4-PE (BeckmanCoulter), CD45RO-ECD (Beckman-Coulter), CD8-PC7 (Becton-Dickinson), CD27-APC (Becton-Dickinson), CD4-FITC (Becton-Dickinson),
HLA-DR-PE (Becton-Dickinson), CD3-ECD (Beckman-Coulter), CD38-PECY5 (Beckman-Coulter), CD25-PC7 (Becton-Dickinson). After incubation, cytolysis of erythrocytes was done by TQ-prep (BeckmanCoulter) and the samples were analyzed by flow cytometry. Immunophenotyping was done with a Navios 10-color flow cytometer (Beckman-Coulter).

Immunological gating strategy was applied for the identification of both the main lymphocyte subpopulation (T, B, and NK) and other subsets, focusing on T-cells maturation and activation (Figure 1). With the extended panel, the following T-cell subpopulations were determined: Naïve and Memory CD4 T-cells expressing CD45RA and CD45R0, respectively; Terminally-differentiated effector and memory T-cells CD45RA+ CD27- and Naïve CD8 T-cells CD45RA+ CD27+; Activated T-cells CD3+ CD38+; Regulatory T-cells CD4+ CD25+; Activated T-cells CD3+ HLA-DR+.

ELISA was used to measure IL6 (Siemens, IMMULITE 1000). Immunonephelometry was used to measure CRP (Siemens, Dimension VISTA). WBC counts were obtained with Sysmex XE-series hematology analyzers (Sysmex, Kobe, Japan).

CRP shows moderate accuracy in prediction of hCA and was not measured in this mother's cohort [11].

\section{Statistics}

Statistical analyses were run using the GraphPad Prism software (v. 5 GraphPad Inc., La Jolla, CA), and the SPSS statistical package (v. 26; IBM, Armonk, NY). The results are expressed as median, and min-max or $\mathrm{n}$ (\%). The data were analyzed using Pearson's chi-squared in the case of
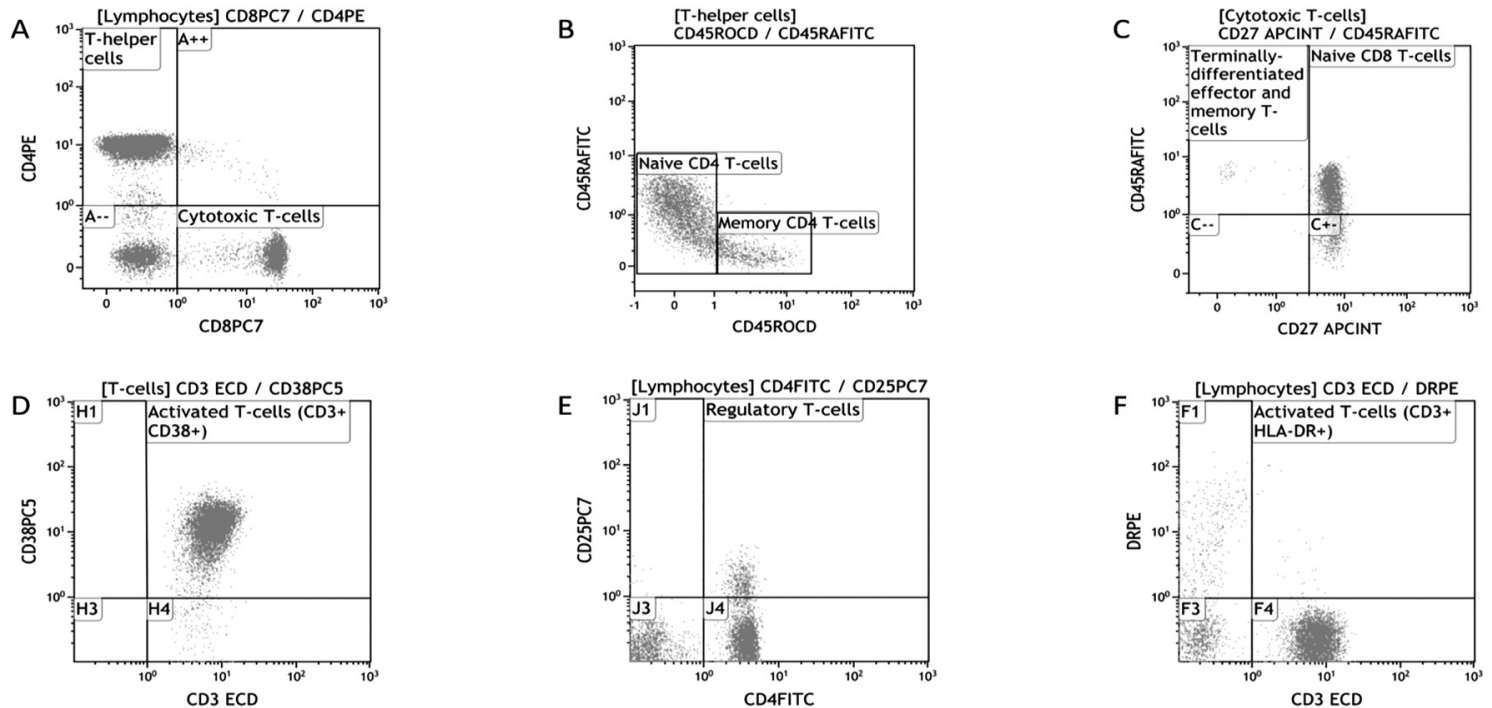

Figure 1: Flow cytometric analysis of T-cell subpopulations in a newborn.

Immunological gating for the identification of the following subsets. T-helper and cytotoxic T-cells expressing CD4 and CD8, respectively (A), naïve and memory CD4 T-cells expressing CD45RA and CD45R0, respectively (B), terminally-differentiated effector and memory T-cells CD45RA+ CD27- and naïve CD8 T-cells CD45RA+ CD27+ (C), activated T-cells CD3+ CD38+ (D), regulatory T-cells CD4+ CD25+ (E), activated T-cells CD3+ HLA-DR+ (F). 
dichotomous variables, and the Mann-Whitney test for continuous variables. Pearson's correlation coefficient was calculated to measure the binomial correlation among variables, and dichotomous logistic regression was used to correlate T-cell subpopulations with gender. A p-value $<0.05$ was always considered as the limit for statistical significance.

\section{Results}

\section{Demographics and clinical data of the newborns and mothers}

The maternal and neonatal demographics and clinical data of our groups of cases and controls are summarized in Table $1 \mathrm{~A}, \mathrm{~B}$.

\section{Effects of pPROM on mothers' and their newborns' laboratory tests}

Table 2 summarizes the distribution of lymphocyte subsets and inflammation markers in newborn cases and controls (Table 2A); and in mothers with and without pPROM (Table 2B).

$\mathrm{T}$-helper cells $(\mathrm{p}=0.02)$, the $\mathrm{CD} 4 / \mathrm{CD} 8$ ratio $(\mathrm{p}=0.02)$, and memory CD4 T-cells $(\mathrm{p}=0.019)$ were higher in pPROM-newborns than in control newborns, while NK cells $(\mathrm{p}=0.01)$, and naïve T-helper (CD4) cells $(\mathrm{p}=0.05)$ were lower in the former than in the latter.

Women who did not experience pPROM had higher levels of non-major histocompatibility complex (MHC)restricted cytotoxic cells $(\mathrm{p}=0.01)$ than the $\mathrm{pPROM}$ mothers.

Figure 1 shows an example of the flow cytometric analysis of T-cell subpopulations in a newborn.

\section{Predictors of neonatal morbidity and maternal hCA}

Table 3A-C shows the distributions of the newborns' lymphocyte subsets and inflammation markers in relation to neonatal BPD and sepsis, and maternal hCA.

Table 1A: Neonatal demographics and clinical data.

\begin{tabular}{|c|c|c|c|}
\hline Data & $\begin{array}{r}\text { pPROM-newborns } \\
\text { Median (min-max), n (\%) }\end{array}$ & $\begin{array}{r}\text { No-pPROM-newborns } \\
\text { Median (min-max), n (\%) }\end{array}$ & $\begin{array}{l}\text { Significance } \\
\text { p-Value }\end{array}$ \\
\hline $\mathrm{n}$ & 23 & 14 & \\
\hline Caucasian & $22(95.7 \%)$ & $13(92.9 \%)$ & ns \\
\hline Asian & $1(4.3 \%)$ & 1 (7.1\%) & ns \\
\hline Male & $15(65.2 \%)$ & $10(71.4 \%)$ & ns \\
\hline Female & $8(34.8 \%)$ & $4(28.6 \%)$ & ns \\
\hline $\mathrm{GA}$, weeks ${ }^{\text {days } / 7}$ & $30^{4 / 7}\left(23^{0 / 7}-34^{6 / 7}\right)$ & $30^{6}\left(23^{6 / 7}-35^{5 / 7}\right)$ & ns \\
\hline BW, g & $1,380(500-2,280)$ & $1,400(590-2,740)$ & ns \\
\hline Antenatal steroids & $21(91.3 \%)$ & $12(85.7 \%)$ & ns \\
\hline Cord blood $\mathrm{pH}<7.25$ & $7(30.4 \%)$ & $2(14.3 \%)$ & ns \\
\hline Head circumference, $\mathrm{cm}$ & $29.3(20.0-33.5)$ & $28.5(22.0-34.0)$ & ns \\
\hline Length, $\mathrm{cm}$ & $41.0(29.0-46.0)$ & $40.3(28.0-48.0)$ & ns \\
\hline \multicolumn{4}{|l|}{ Apgar score } \\
\hline $1^{\prime}$ & $6(2-9)$ & $7(2-9)$ & ns \\
\hline $5^{\prime}$ & $7(4-10)$ & $8(6-9)$ & ns \\
\hline $10^{\prime}$ & $8(7-10)$ & $9(8-10)$ & ns \\
\hline RDS & $15(65.2 \%)$ & $10(71.4 \%)$ & ns \\
\hline Inotropes and vasopressors & $5(21.7 \%)$ & $2(14.3 \%)$ & ns \\
\hline Mechanical ventilation & 21 (91.3\%) & $12(85.7 \%)$ & ns \\
\hline Mechanical ventilation, days & $20(0.5-93)$ & $12(0.5-92)$ & ns \\
\hline $\mathrm{FiO}_{2}, \%$ & $21(21-50)$ & $21(21-75)$ & ns \\
\hline Surfactant & 9 (39.1\%) & $7(50 \%)$ & ns \\
\hline BPD & $9(39.1 \%)$ & $5(35.7 \%)$ & ns \\
\hline PDA & $6(26.1 \%)$ & $4(28.6 \%)$ & ns \\
\hline EOS & $13(56.5 \%)$ & $3(21.4 \%)$ & 0.036 \\
\hline IVH & $4(17.4 \%)$ & $2(14.3 \%)$ & ns \\
\hline NEC & $2(8.7 \%)$ & $1(7.1 \%)$ & ns \\
\hline ROP & $3(13 \%)$ & $5(35.7 \%)$ & ns \\
\hline
\end{tabular}

Data are expressed as median (min-max) in the case of continuous variables, or number (\%) in the case of dichotomous variables. GA, gestational age; BW, birth weight; g, grams; RDS, respiratory distress syndrome; BPD, bronchopulmonary dysplasia; EOS, early onset sepsis; PDA, patent ductus arteriosus; IVH, intraventricular hemorrhage; NEC, necrotizing enterocolitis; ROP, retinopathy of prematurity. 
Table 1B: Maternal demographics and clinical data.

\begin{tabular}{lrrr}
\hline Data & $\begin{array}{r}\text { pPROM mothers } \\
\text { Median (min-max), } \mathbf{n}(\%)\end{array}$ & $\begin{array}{r}\text { No-pPROM mothers } \\
\text { Median (min-max), } \mathbf{n}(\%)\end{array}$ & $\begin{array}{c}\text { Significance } \\
\mathbf{p}-\text { Value }\end{array}$ \\
\hline $\mathrm{n}$ & 18 & 11 & $\mathrm{~ns}$ \\
Age, years & $34.0(25-44)$ & $34.0(24-47)$ & $\mathrm{ns}$ \\
Caucasian & $17(94.4 \%)$ & $10(90.9 \%)$ & $\mathrm{ns}$ \\
Asian & $1(5.6 \%)$ & $1(9.1 \%)$ & 0.024 \\
Primiparous & $9(50 \%)$ & $10(90.9 \%)$ & $\mathrm{ns}$ \\
Twin pregnancies & $2(11.1 \%)$ & $2(18.2 \%)$ & $\mathrm{ns}$ \\
Fever (T>37.5 ${ }^{\circ}$ C) & $3(16.7 \%)$ & $0(0 \%)$ & $\mathrm{ns}$ \\
Steroid administration & $15(83.3 \%)$ & $9(81.8 \%)$ & 0.031 \\
Mode of delivery (CS) & $12(66.7 \%)$ & $11(100 \%)$ & 0.001 \\
hCA & $11(61.1 \%)$ & $0(0 \%)$ & $\mathrm{ns}$ \\
Placental weight, g & $320(185-901)$ & $420(150-830)$ & \\
\hline
\end{tabular}

Data are expressed as median ( $\min -\max$ ) in the case of continuous variables; or number (\%) in the case of dichotomous variables. CS, cesarean section; hCA, histologically-confirmed chorioamnionitis; g, grams.

Table 2A: Distribution of lymphocyte subsets and inflammation markers in pPROM and no-pPROM-newborn.

\begin{tabular}{lrrl}
\hline T-cell phenotypes & \multicolumn{2}{c}{ Median (min-max) } & p-Value (Mann-Whitney test) \\
\cline { 2 - 3 } & pPROM-newborn & No-pPROM-newborn & \\
\hline T-cells, \% & $77.0(49.0-95.0)$ & $76.0(50.0-82.0)$ & $\mathrm{ns}$ \\
T-helper cells, \% & $62.0(35.0-79.0)$ & $54.0(34.0-62.0)$ & 0.02 \\
Absolute T-helper cell count, $\times 10^{9} / \mathrm{L}$ & $2.40(0.45-4.04)$ & $1.26(0.49-2.48)$ & 0.04 \\
Cytotoxic T-cells, \% & $16.0(11.0-33.0)$ & $19.0(15.0-27.0)$ & $\mathrm{ns}$ \\
B and pre-B cells, \% & $12.0(2.0-30.0)$ & $9.0(6.0-29.0)$ & $\mathrm{ns}$ \\
NK cells, \% & $6.0(2.0-29.0)$ & $14.0(5.0-35.0)$ & 0.01 \\
Non-MHC-restricted cytotoxic cells, \% & $0(0.0-2.0)$ & $0.0(0.0-1.0)$ & $\mathrm{ns}$ \\
CD4/CD8 ratio & $3.51(1.56-6.39)$ & $2.30(1.29-3.67)$ & 0.02 \\
Naïve CD4 T-cells, \% & $90.0(78.0-98.0)$ & $93.5(82.0-100.0)$ & 0.05 \\
Memory CD4 T-cells, \% & $10.0(2.0-22.0)$ & $5.0(0.0-15.0)$ & 0.019 \\
Naïve CD8 T-cells, \% & $85.0(52.0-98.0)$ & $86.0(76.0-97.0)$ & $\mathrm{ns}$ \\
Terminally-differentiated effector and memory T-cells, \% & $2.6(0.3-13.0)$ & $3.0(0.4-11.0)$ & $\mathrm{ns}$ \\
Activated T-cells (CD3+ CD38+), \% & $96.0(90.0-98.0)$ & $94.0(80.0-97.0)$ & $\mathrm{ns}$ \\
Regulatory T-cells, \% & $3.95(0.4-7.0)$ & $3.9(3.0-6.0)$ & $\mathrm{ns}$ \\
Activated T-cells (CD3+ HLA-DR+), \% & $0.4(0.1-0.7)$ & $0.2(0.1-0.5)$ & $\mathrm{ns}$ \\
CRP, mg/L & $2.9(1.5-31.0)$ & $2.9(1.6-11.0)$ & $\mathrm{ns}$ \\
IL-6, ng/L & $10.1(2.9-5,000.0)$ & $91.5(2.0-3,180.0)$ & $\mathrm{ns}$ \\
Leukocytes, $\times 10^{9} / \mathrm{L}$ & $8.6(1.7-40.1)$ & $10.1(3.7-15.2)$ & $\mathrm{ns}$ \\
\hline
\end{tabular}

Data are expressed as median (min-max). NK cells, natural killer cells; CD4, T-helper cells; CD8, cytotoxic T-cells; CRP, C-reactive protein; IL-6, interleukin-6; MHC, major histocompatibility complex.

The newborns who went on to develop BPD had fewer naïve CD8 T-cells $(\mathrm{p}=0.0009)$ at birth than those who did not.

Sepsis caused an increase in T-helper lymphocytes in the affected newborns compared with controls $(p=0.04)$ and also - as expected - in their CRP levels $(p=0.009)$. The newborns of mothers who had hCA showed an increase in T-helper lymphocytes compared with controls $(\mathrm{p}=0.02)$.

\section{Effect of pPROM latency period}

Table 4 summarizes the effect of a latency period between the diagnosis pPROM and delivery of more or less than 3 weeks on T-cell phenotypes. A latency $\leq 3$ weeks correlated with a stronger expression of naïve CD4 T-cells, while a longer latency ( $>3$ weeks) coincided with an increase in memory CD4 T-cells. The level of leukocytes also rose with a longer latency, but the difference was not statistically significant $(\mathrm{p}=0.06)$. 
Table 2B: Distribution of lymphocyte subsets and inflammation markers in mothers with and without pPROM.

\begin{tabular}{|c|c|c|c|}
\hline \multirow[t]{2}{*}{ T-cell phenotypes } & \multicolumn{2}{|c|}{ Median (min-max) } & \multirow[t]{2}{*}{ p-Value (Mann-Whitney test) } \\
\hline & pPROM mothers & No-pPROM mothers & \\
\hline T-cells, \% & $80.5(69.0-87.0)$ & $82.0(66.0-89.0)$ & ns \\
\hline T-helper cells, \% & $50.0(34.0-62.0)$ & $50.0(38.0-64.0)$ & ns \\
\hline Absolute T-helper cell count, $\times 10^{9} / \mathrm{L}$ & $0.98(0.44-1.63)$ & $0.98(0.69-1.94)$ & ns \\
\hline Cytotoxic T-cells, \% & $29.0(15.0-43.0)$ & $27.0(17.0-41.0)$ & ns \\
\hline$B$ and pre-B cells, $\%$ & $11.5(4.0-25.0)$ & $13.0(6.0-26.0)$ & ns \\
\hline NK cells, \% & $6.5(3.0-16.0)$ & $4.0(3.0-10.0)$ & ns \\
\hline Non-MHC-restricted cytotoxic cells, \% & $3.5(1.0-7.0)$ & $5.0(2.0-15.0)$ & 0.01 \\
\hline CD4/CD8 ratio & $1.73(1.01-4.06)$ & $1.72(0.94-3.14)$ & ns \\
\hline Naïve CD4 T-cells, \% & $45.5(30.0-60.0)$ & $46.0(32.0-71.0)$ & ns \\
\hline Memory CD4 T-cells, \% & $54.5(40.0-70.0)$ & $54.0(29.0-68.0)$ & ns \\
\hline Naïve CD8 T-cells, \% & $53.0(17.3-77.0)$ & $61.5(30.0-80.0)$ & ns \\
\hline Terminally-differentiated effector and memory T-cells, \% & $9.4(2.3-40.0)$ & $12.5(2.0-17.0)$ & ns \\
\hline Activated T-cells (CD3+ CD38+), \% & $44.0(8.0-82.0)$ & $45.5(22.0-80.0)$ & ns \\
\hline Regulatory T-cells, \% & $1.1(0.0-3.9)$ & $1.5(0.1-5.7)$ & ns \\
\hline Activated T-cells (CD3+ HLA-DR+), \% & $5.0(1.4-13.0)$ & $3.0(1.5-8.7)$ & ns \\
\hline IL-6, ng/L & $12.2(3.6-64.4)$ & $11.4(2.0-30.3)$ & ns \\
\hline Leukocytes, $\times 10^{9} / \mathrm{L}$ & $13.6(8.6-31.7)$ & $10.9(7.3-18.4)$ & ns \\
\hline
\end{tabular}

Data are expressed as median (min-max). NK cells, natural killer cells; CD4, T-helper cells; CD8, cytotoxic T-cells; IL-6, interleukin-6; MHC, major histocompatibility complex.

Table 3A: Distribution of neonatal lymphocyte subsets and inflammation markers and BPD.

\begin{tabular}{|c|c|c|c|}
\hline \multirow[t]{2}{*}{ T-cell phenotypes } & \multicolumn{2}{|c|}{ Median (min-max) } & \multirow[t]{2}{*}{ p-Value (Mann-Whitney test } \\
\hline & BPD $(n=9)$ & No-BPD $(n=14)$ & \\
\hline T-cells, \% & $73.5(49.0-93.0)$ & $79.0(52.0-95.0)$ & ns \\
\hline T-helper cells, \% & $56.0(35.0-79.0)$ & $60.5(34.0-77.0)$ & ns \\
\hline Absolute T-helper cell count, $\times 10^{9} / \mathrm{L}$ & $1.78(0.48-4.04)$ & $1.81(0.45-3.84)$ & ns \\
\hline Cytotoxic T-cells, \% & $15.5(11.0-26.0)$ & $18.0(13.0-33.0)$ & ns \\
\hline B and pre-B cells, $\%$ & $13.0(4.0-29.0)$ & $9.0(2.0-30.0)$ & ns \\
\hline NK cells, \% & $12.5(2.0-29.0)$ & $6.5(2.0-35.0)$ & ns \\
\hline Non-MHC-restricted cytotoxic cells, \% & $0.0(0.0-2.0)$ & $0.0(0.0-1.0)$ & ns \\
\hline CD4/CD8 ratio & $3.28(1.58-6.39)$ & $3.12(1.56-4.93)$ & ns \\
\hline Naïve CD4 T-cells, \% & $90.0(79.0-98.0)$ & $90.0(78.0-100.0)$ & ns \\
\hline Memory CD4 T-cells, \% & $10.0(2.0-21.0)$ & $10.0(0.0-22.0)$ & ns \\
\hline Naïve CD8 T-cells, \% & $80.5(52.0-91.0)$ & $87.0(79.0-98.0)$ & 0.0009 \\
\hline Terminally-differentiated effector and memory T-cells, \% & $5.0(0.6-13.0)$ & $2.4(0.3-9.0)$ & ns \\
\hline Activated T-cells (CD3+ CD38+), \% & $96.0(90.0-98.0)$ & $95.0(80.0-98.0)$ & ns \\
\hline Regulatory T-cells, \% & $4.0(1.5-7.0)$ & $3.9(0.4-6.7)$ & ns \\
\hline Activated T-cells (CD3+ HLA-DR+), \% & $0.2(0.1-0.7)$ & $0.3(0.1-0.5)$ & ns \\
\hline $\mathrm{CRP}, \mathrm{mg} / \mathrm{L}$ & $2.9(1.5-22.3)$ & $2.9(1.6-31.0)$ & ns \\
\hline IL-6, ng/L & $66.8(6.5-3,180.0)$ & $8.9(2.0-5,000.0)$ & ns \\
\hline Leukocytes, $\times 10^{9} / \mathrm{L}$ & $10.3(1.7-40.6)$ & $9.3(2.1-27.5)$ & ns \\
\hline
\end{tabular}

Data are expressed as median (min-max). NK cells, natural killer cells; CD4, T-helper cells; CD8, cytotoxic T-cells; CRP, C-reactive protein; IL-6, interleukin-6; MHC, major histocompatibility complex.

Among the mothers in the pPROM group, a latency of more or less than 3 weeks made no difference to their T-cell phenotypes.

\section{Gender and T-cell phenotypes}

Logistic regression revealed no differences between the T-cell immunophenotypes of the male and female newborn. 
Table 3B: Distribution of neonatal lymphocyte subsets and inflammation markers and sepsis.

\begin{tabular}{|c|c|c|c|}
\hline \multirow[t]{2}{*}{ T-cell phenotypes } & \multicolumn{2}{|c|}{ Median (min-max) } & \multirow[t]{2}{*}{ p-Value (Mann-Whitney test) } \\
\hline & $\operatorname{EOS}(n=13)$ & No-EOS $(n=10)$ & \\
\hline T-cells, \% & $82.5(50.0-95.0)$ & $75.0(49.0-89.0)$ & ns \\
\hline T-helper cells, \% & $68.5(35.0-79.0)$ & $56.0(34.0-74.0)$ & ns \\
\hline Absolute T-helper cell count, $\times 10^{9} / \mathrm{L}$ & $2.59(0.49-4.04)$ & $1.50(0.45-3.84)$ & 0.04 \\
\hline Cytotoxic T-cells, \% & $16.0(11.0-24.0)$ & $18.0(12.0-33.0)$ & ns \\
\hline$B$ and pre-B cells, $\%$ & $10.5(2.0-29.0)$ & $10.5(6.0-30.0)$ & ns \\
\hline NK cells, \% & $5.0(2.0-2.0)$ & $9.5(2.0-35.0)$ & ns \\
\hline Non-MHC-restricted cytotoxic cells, \% & $0.0(0.0-1.0)$ & $0.0(0.0-2.0)$ & ns \\
\hline CD4/CD8 ratio & $3.90(2.23-6.39)$ & $3.08(1.56-4.93)$ & ns \\
\hline Naïve CD4 T-cells, \% & $90.0(78.0-95.0)$ & $91.0(79.0-100.0)$ & ns \\
\hline Memory CD4 T-cells, \% & $10.0(5.0-22.0)$ & $9.0(0.0-21.0)$ & ns \\
\hline Naïve CD8 T-cells, \% & $83.0(65.0-91.0)$ & $86.5(70.0-98.0)$ & ns \\
\hline Terminally-differentiated effector and memory T-cells, \% & $3.0(0.3-13.0)$ & $3.0(0.4-11.0)$ & ns \\
\hline Activated T-cells (CD3+CD38+), \% & $96.0(90.0-98.0)$ & $95.0(80.0-98.0)$ & ns \\
\hline Regulatory T-cells, \% & $4.3(1.5-6.3)$ & $3.8(0.4-7.0)$ & ns \\
\hline Activated T-cells (CD3+ HLA-DR+), \% & $0.4(0.2-0.7)$ & $0.3(0.1-0.6)$ & ns \\
\hline CRP, mg/L & $9.2(1.6-31.0)$ & $2.9(2.9-6.3)$ & 0.009 \\
\hline IL-6, ng/L & $32.7(10.1-5,000.0)$ & $21.9(2.0-272.0)$ & ns \\
\hline Leukocytes, $\times 10^{9} / \mathrm{L}$ & $12.7(2.1-40.6)$ & $8.7(3.7-27.5)$ & ns \\
\hline
\end{tabular}

Data are expressed as median (min-max). NK cells, natural killer cells; CD4, T-helper cells; CD8, cytotoxic T-cells; CRP, C-reactive protein; IL-6, interleukin-6; EOS, early onset sepsis; MHC, major histocompatibility complex.

Table 3C: Distribution of neonatal lymphocyte subsets and inflammation markers and maternal hCA.

\begin{tabular}{|c|c|c|c|}
\hline \multirow[t]{2}{*}{ T-cell phenotypes } & \multicolumn{2}{|c|}{ Median (min-max) } & \multirow[t]{2}{*}{ p-Value (Mann-Whitney test) } \\
\hline & hCA $(n=11)$ & No-hCA $(n=7)$ & \\
\hline T-cells, \% & $83.0(49.0-95.0)$ & $75.0(50.0-89.0)$ & ns \\
\hline T-helper cells, \% & $63.0(35.0-79.0)$ & $56.5(34.0-74.0)$ & ns \\
\hline Absolute T-helper cell count, $\times 10^{9} / \mathrm{L}$ & $2.59(0.48-4.04)$ & $1.29(0.45-3.84)$ & 0.02 \\
\hline Cytotoxic T-cells, \% & $16.0(11.0-33.0)$ & $19.5(13.0-27.0)$ & ns \\
\hline$B$ and pre-B cells, $\%$ & $9.0(2.0-20.0)$ & $13.0(6.0-30.0)$ & ns \\
\hline NK cells, \% & $6.0(2.0-29.0)$ & $10.0(2.0-35.0)$ & ns \\
\hline Non-MHC-restricted cytotoxic cells, \% & $0.0(0.0-1.0)$ & $0.0(0.0-2.0)$ & ns \\
\hline CD4/CD8 ratio & $3.63(1.56-6.39)$ & $2.94(1.29-4.93)$ & ns \\
\hline Naïve CD4 T-cells, \% & $87.5(78.0-98.0)$ & $91.0(79.0-100.0)$ & ns \\
\hline Memory CD4 T-cells, \% & $12.5(2.0-22.0)$ & $10.0(0.0-21.0)$ & ns \\
\hline Naïve CD8 T-cells, \% & $82.5(52.0-98.0)$ & $86.0(74.0-97.0)$ & ns \\
\hline Terminally-differentiated effector and memory T-cells, \% & $3.7(0.3-13.0)$ & $3.0(0.4-11.0)$ & ns \\
\hline Activated T-cells (CD3+ CD38+), \% & $96.5(90.0-98.0)$ & $95.5(80.0-98.0)$ & ns \\
\hline Regulatory T-cells, \% & $4.3(0.4-6.7)$ & $3.9(2.7-7.0)$ & ns \\
\hline Activated T-cells (CD3+ HLA-DR+), \% & $0.3(0.1-0.7)$ & $0.3(0.1-0.6)$ & ns \\
\hline $\mathrm{CRP}, \mathrm{mg} / \mathrm{L}$ & $2.9(1.5-31.0)$ & $2.9(1.6-11.0)$ & ns \\
\hline IL-6, ng/L & $11.1(6.5-5,000.0)$ & $25.2(2.0-3,180.0)$ & ns \\
\hline Leukocytes, $\times 10^{9} / \mathrm{L}$ & $11.5(1.7-40.6)$ & $9.7(3.7-18.2)$ & ns \\
\hline
\end{tabular}

Data are expressed as median (min-max). NK cells, natural killer cells; CD4, T-helper cells; CD8, cytotoxic T-cells; CRP, C-reactive protein; IL-6, interleukin-6; MHC, major histocompatibility complex.

\section{GA/BW and laboratory data}

Table 5 shows Pearson's correlations: with increasing GA there was a gradual reduction in leukocyte $(\mathrm{p}=0.046)$. The results showed negative correlations between white blood cell counts, cytotoxic T-cells ( $\mathrm{p}=0.009)$, and regulatory T-cells $(\mathrm{p}=0.016)$, and positive correlations between leukocyte counts, absolute T-helper cell counts $(\mathrm{p}=0.002)$, and CD4/CD8 ratios $(\mathrm{p}=0.004)$. 
Table 4: Distribution of newborns' lymphocyte subsets and inflammation markers by latency period after pPROM.

\begin{tabular}{|c|c|c|c|}
\hline \multirow[t]{2}{*}{ T-cell phenotypes } & \multicolumn{2}{|c|}{ Median (min-max) } & \multirow[t]{2}{*}{ p-Value (Mann-Whitney test) } \\
\hline & Latency $\leq 3$ weeks & Latency >3 weeks & \\
\hline T-cells, \% & $82(49.0-95.0)$ & $70.0(60.0-89.0)$ & ns \\
\hline T-helper cells, \% & $62(35.0-79.0)$ & $54(49.0-69.0)$ & ns \\
\hline Absolute T-helper cell count, $\times 10^{9} / \mathrm{L}$ & $2.40(0.46-4.04)$ & $2.44(1.66-3.84)$ & ns \\
\hline Cytotoxic T-cells, \% & $16(11.0-33.0)$ & $16.5(12.0-20)$ & ns \\
\hline $\mathrm{B}$ and pre-B cells, $\%$ & $12(2.0-30.0)$ & $11.0(9.0-14.0)$ & ns \\
\hline NK cells, \% & $6(2.0-29.0)$ & $18.0(2.0-26.0)$ & ns \\
\hline Non-MHC-restricted cytotoxic cells, \% & $0.0(0.0-2.0)$ & $0.0(0.0-0.0)$ & ns \\
\hline CD4/CD8 ratio & $3.63(1.56-6.39)$ & $3.51(3.11-4.18)$ & ns \\
\hline Naïve CD4 T-cells, \% & $90.0(78.0-98.0)$ & $83.0(79.0-85.0)$ & 0.04 \\
\hline Memory CD4 T-cells, \% & $10.0(2.0-22.0)$ & $17.0(15.0-21.0)$ & 0.04 \\
\hline Naïve CD8 T-cells, \% & $85.0(52.0-98.0)$ & $83.0(81.0-85.0)$ & ns \\
\hline Terminally-differentiated effector and memory T-cells, \% & $2.5(0.3-13.0)$ & $5.0(0.6-5.5)$ & ns \\
\hline Activated T-cells (CD3+ CD38+), \% & $96.0(90.0-98.0)$ & $95.0(92.0-96.4)$ & ns \\
\hline Regulatory T-cells, \% & $4.0(1.5-7.0)$ & $3.6(0.4-5.2)$ & ns \\
\hline Activated T-cells (CD3+ HLA-DR+), \% & $0.3(0.1-0.7)$ & $0.46(0.4-0.5)$ & ns \\
\hline $\mathrm{CRP}, \mathrm{mg} / \mathrm{L}$ & $2.9(1.5-31.0)$ & $2.9(2.9-2.9)$ & ns \\
\hline IL-6, ng/L & $11.1(2.9-5,000.0)$ & $5.25(3.4-7.1)$ & ns \\
\hline Leukocytes, $\times 10^{9} / \mathrm{L}$ & $8.2(1.7-40.6)$ & $19.35(7.71-27.5)$ & 0.068 \\
\hline
\end{tabular}

Data are expressed as median (min-max). NK cells, natural killer cells; CD4, T-helper cells; CD8, cytotoxic T-cells; CRP, C-reactive protein; IL-6, interleukin-6; MHC, major histocompatibility complex.

Table 5: Pearson's correlations.

\begin{tabular}{|c|c|c|c|c|}
\hline & & GA, week & BW, gram & Leukocytes, $\times 10^{9} / \mathrm{L}$ \\
\hline \multirow[t]{2}{*}{ GA, week } & Pearson's $r$ & 1 & $0.945^{\mathrm{b}}$ & $-0.330^{\mathrm{a}}$ \\
\hline & Two-tailed significance & & 0 & 0.046 \\
\hline \multirow[t]{2}{*}{ BW, g } & Pearson's $r$ & $0.945^{\mathrm{b}}$ & 1 & -0.29 \\
\hline & Two-tailed significance & 0 & & 0.081 \\
\hline \multirow[t]{2}{*}{ Absolute T-helper cell count, $\times 10^{9} / \mathrm{L}$} & Pearson's r & -0.017 & 0.089 & $0.495^{b}$ \\
\hline & Two-tailed significance & 0.923 & 0.611 & 0.002 \\
\hline \multirow[t]{2}{*}{ Cytotoxic T-cells, \% } & Pearson's r & 0.196 & 0.165 & $-0.427^{b}$ \\
\hline & Two-tailed significance & 0.252 & 0.336 & 0.009 \\
\hline \multirow[t]{2}{*}{ CD4/CD8 ratio } & Pearson's r & -0.099 & -0.042 & $0.468^{b}$ \\
\hline & Two-tailed significance & 0.568 & 0.806 & 0.004 \\
\hline \multirow[t]{2}{*}{ Regulatory T-cells, \% } & Pearson's r & 0.144 & 0.12 & $-0.417^{\mathrm{a}}$ \\
\hline & Two-tailed significance & 0.424 & 0.507 & 0.016 \\
\hline \multirow[t]{2}{*}{ Leukocytes, $\times 10^{9} / \mathrm{L}$} & Pearson's r & $-0.330^{\mathrm{a}}$ & -0.29 & 1 \\
\hline & Two-tailed significance & 0.046 & 0.081 & \\
\hline
\end{tabular}

Data are Pearson's $r$ and $p$-value: ${ }^{a}$ significance level <0.05; ${ }^{b}$ significance level <0.01. GA, gestational age; BW, birth weight; g, grams.

\section{Discussion}

\section{Effect of pPROM on the mothers' and newborns' T-cell phenotype}

This study found pPROM associated with an increase in the proportion of neonatal T-helper cells, the $\mathrm{CD} 4 / \mathrm{CD} 8$ ratio and memory CD4 T-cells, and a decrease in naïve CD4 T-cells. In our pPROM group, we also found a higher incidence of sepsis in the newborn, and a higher incidence of hCA in the mothers [12]. The mothers' T-cell immunophenotype did not express a particular distribution, apart from the nonMCH-restricted cytotoxic cells (\%), which were lower in pPROM cases than in controls.

All these results would suggest an early antigenic stimulation in the newborn delivered after pPROM that may begin already in utero, and that stimulates an immunological memory. The increase in T-helper subpopulations may also be a consequence of this stimulatory effect [13]. Whether this early antigenic stimulation is due to an infection occurring 
during or after the pPROM [4], or secondary to a cytokine cascade triggered by pPROM per se is not known [7]. Instead of an activation of the maternal lymphocyte system, there is a much more evident early immunological response in the newborn after pPROM.

The greater prevalence of hCA in our pPROM group would support the hypothesis of an infection-driven immune stimulation. In other words, intrauterine infection prompts an immune stimulation [4], and is associated with a higher risk of hCA and sepsis, after birth occurred under pPROM $[1,14]$. This condition could support the conversion of naïve T-cells into memory T-cells, with an overall increase in the proportion of the latter.

If, when pPROM occurs, fetuses react to infection and thus have more memory CD4 T-lymphocytes at birth, then their immune system will mature early. If their immune system is activated against the mother, this would conceivably trigger preterm birth, and pave the way to the infants' future immune tolerance.

Newborns experiencing pPROM were characterized by a rise in T-helper lines and memory CD4 T-cells, by comparison with those unaffected by any pPROM. The latter showed a surprising (but statistically insignificant) rise in IL-6. A lower proportion of NK cells in pPROM-newborn would increase the risk of subsequent infection in very preterm infants $[15,16]$. NK cells are a component of the innate immune system. They play a major part in the host's rejection response to infectious diseases. They typically detect the MH complex present on infected cell surfaces, trigger cytokine release, and cause the death of cells infected by viruses via lysis and apoptosis. NK cells are unique in their ability to recognize and kill stressed cells in the absence of antibodies and MHC, enabling a steady immune reaction.

The maternal data reported here do not seem to support the theory of a passage of maternal immune cells to the newborn. The maternal lymphocyte profile that we identified revealed no crucial differences in the women experiencing pPROM. A limitation of this study lies in that we did not consider T-helper 17 cells, which might have clarified maternal involvement from an inflammatory/infectious standpoint, and that we did not use intracytoplasmic Foxp3 for a more reliable identification of regulatory T-cells. pPROM mothers had lower levels of immunomodulatory non-MHC-restricted cytotoxic cells, which support the attachment of the embryo and the maturation of the placenta. In early pregnancy, progesterone drives the transformation of uterine stroma into decidual cells - a mechanism also regulated by chemokines, enzymes/proteases and T-cells, which may regulate trophoblast proliferation [17]. The decrease in the cytotoxic lineage may also be the sign of an adaptive immune tolerance towards the fetus, especially in pPROM; this would be an adaptation of the innate immune response to the adaptive $[18,19]$.

It may be that T-cell plasticity towards cytotoxicity is reduced in the case of pPROM [20], and this would suggest the specific use of T-cells lines for therapeutic purposes after phenotype polarization or stimulation [21].

\section{Predictors of neonatal morbidity and maternal hCA}

The newborns who went on to develop BPD were characterized by lower numbers of naïve CD8 T-cells than in controls. BPD is a GA-correlated morbidity [22-24], especially affecting very premature newborns. The period immediately after delivery is characterized by a low number of T-cells, which increase after antigenic stimulation. It is worth noting, however, that it is impossible to tell whether the lower number of naïve CD8 T-lymphocytes at birth is predictive of BPD or merely due to a younger GA. Further studies will be needed to clarify this issue.

In cases of hCA and sepsis, there should be an increase in the number of T-helper lymphocytes due to the presence of infection. hCA is a known risk factor for sepsis and, during intrauterine infection, there is an increase in the number of T-helper cells in the affected newborns $[1,4,14]$.

\section{Effect of latency period between PPROM and delivery}

We found fewer naïve T-cells, more memory CD4 T-cells and more leukocytes in newborns delivered with a prolonged latency after pPROM. Fetuses staying in utero for longer than 3 weeks were able to develop a neonatal adaptive immune response and an early immunological reaction to foreign antigens $[15,16]$. This can be explained by their prolonged antigenic stimulation due to intra-amniotic contamination [9], which causes early maturation of the newborn's immune system. It would be interesting to ascertain whether this in utero antigenic stimulation is led by pathogens or by antigen's surface of maternal cells.

Our mothers' T-cells did not appear to change after a latency of more than 3 weeks. This result is in contrast with the reported increase in anti-fetal HLA and T-cell responsiveness in mothers experiencing pPROM [7].

\section{GA, BW, and laboratory data}

As previously reported [25], we found an inverse correlation between blood leukocytes and GA or BW in our overall 
newborns, and the same was true of the cytotoxic T-cells and regulatory T-cells. On the other hand, T-helper cells (and the CD4/CD8 ratio) increased with GA or BW. A recent investigation [25] also showed that neutrophils in tracheal aspirates correlated with $\mathrm{BW}$ and $\mathrm{GA}$, give that immunological maturation also occurs for lung inflammatory cells. Many clinical factors may influence leukocyte levels, however, including mode of delivery [4, 26-28], eclampsia, antenatal steroids, GA and BW, as well as phenotype and T-cell levels.

Our study is a single center study with a small sample size: since it is difficult to obtain parallel data from admitted mother/newborn couple (which is the main criterion of inclusion), this investigation is a pilot study with a future perspective of data validation. Since pre-eclamptic women may show an abnormal activation of the immune system and may be affected by a rise in inflammatory markers the inclusion of a single case in the controls represents a bias in our study $[29,30]$.

A further limitation of our study lies in that we did not measure chemokines or cytokines, though this is quite difficult to do in premature infants and in their blood samples. Our correlation matrices seem to support some sort of organized and connected T-cell action, with an interplay of messengers or shuttling organelles underlying overall lymphocyte function, rather than a specific or individualized cell line effect.

\section{Conclusions}

Our most intriguing results concern the lower levels of naïve CD4 T-lymphocytes, and higher levels of memory CD4 T-cells in pPROM-newborns, indicating a more protracted antigenic stimulation than in controls. This was clearly evident and considerable, especially for longer latency periods between pPROM and delivery. Further studies will be needed to clarify the origin of this antigenic stimulation and the developmental patterns of the $\mathrm{T}$ phenotype associated with preterm birth. It will also be important to further study the role of low levels of naïve CD8 T-cells in BPD, and of high levels of T-helper cells in neonatal early onset sepsis.

Acknowledgments: All the patients and their neonates. Research funding: None declared.

Author contributions: All authors have accepted responsibility for the entire content of this manuscript and approved its submission.

Competing interests: Authors state no conflict of interest. Informed consent: Informed consent was obtained from all individuals included in this study.
Ethical approval: The local Institutional Review Board deemed the study exempt from review.

\section{References}

1. Mercer BM. Preterm premature rupture of the membranes: current approaches to evaluation and management. Obstet Gynecol Clin N Am 2005;32:411.

2. Test G, Levy A, Wiznitzer A, Mazor M, Holcberg G, Zlotnik A, et al. Factors affecting the latency period in patients with preterm premature rupture of membranes. Arch Gynecol Obstet 2011; 283:707-10.

3. Cho HY, Jung I, Kwon JY, Kim SJ, Park YW, Kim YH. The Delta Neutrophil Index as a predictive marker of histological chorioamnionitis in patients with preterm premature rupture of membranes: a retrospective study. PloS One 2017;12:e0173382.

4. Melville JM, Moss TJM. The immune consequences of preterm birth. Front Neurosci 2013;7:79.

5. Tosato F, Bucciol G, Pantano G, Putti MC, Sanzari MC, Basso G, et al. Lymphocytes subsets reference values in childhood. Cytometry 2015;87:81-5.

6. Kan B, Razzaghian HR, Lavoie PM. An immunological perspective on neonatal sepsis. Trends Mol Med 2016;22:292.

7. Steinborn A, Schmitt E, Stein Y, Klee A, Gonser M, Seifried E, et al. Prolonged preterm rupture of fetal membranes, a consequence of an increased maternal anti-fetal T cell responsiveness. Pediatr Res 2005;58:648-53.

8. Redline RW. Classification of placental lesions. Am J Obstet Gynecol 2015;213(4 Suppl):S21-8.

9. Romero R, Gomez-Lopez N, Winters AD, Jung E, Shaman M, Bieda $J$, et al. Evidence that intra-amniotic infections are often the result of an ascending invasion - a molecular microbiological study. J Perinat Med 2019;47:915-31.

10. American Academy of Pediatrics. Group B streptococcal infections. In: Kimberlin DW, Brady MT, Jackson MA, Long SS, editors. Red book: 2018 report of the Committee on Infectious Diseases, 31st ed. Itasca, IL: American Academy of Pediatrics; 2018:762 p.

11. Asadi N, Faraji A, Keshavarzi A, Akbarzadeh-Jahromi M, Yoosefi S. Predictive value of procalcitonin, C-reactive protein, and white blood cells for chorioamnionitis among women with preterm premature rupture of membranes. Int J Gynaecol Obstet 2019; 147:83-8.

12. Al-lawama M, AlZaatreh A, Elrajabi R, Abdelhamid S, Badran E. Prolonged rupture of membranes, neonatal outcomes and management guidelines. J Clin Med Res 2019;11:360-66.

13. Huenecke S, Fryns E, Wittekindt B, Buxmann H, Königs C, Quaiser $A$, et al. Percentiles of lymphocyte subsets in preterm infants according to gestational age compared to children and adolescents. Scand J Immunol 2016;84:291-98.

14. Randis TM, Rice MM, Myatt L, Tita ATN, Leveno KJ, Reddy UM, et al. Incidence of early-onset sepsis in infants born to women with clinical chorioamnionitis. J Perinat Med 2018;46:926-33.

15. Ma L, Chen R, Liu F, Li Y, Wu Z, Zhong W, et al. Reduced NK cell percentage at birth is associated with late onset infection in very preterm neonates. Scand J Immunol 2014;80:50-6. 
16. Amatuni GS, Sciortino S, Currier RJ, Naides SJ, Church JA, Puck JM. Reference intervals for lymphocyte subsets in preterm and term neonates without immune defects. J Allergy Clin Immunol 2019; 144:1674-83.

17. Cheon YP, DeMayo FJ, Bagchi MK, Bagchi IC. Induction of cytotoxic T-lymphocyte antigen-2 $\beta$, a cysteine protease inhibitor in decidua: a potential regulator of embryo implantation. J Biol Chem 2004;279:10357-63.

18. Moser B, Eberl M. $ү \delta T$ cells: novel initiators of adaptive immunity. Immunol Rev 2007;215:89-102.

19. Tyler CJ, Doherty DG, Moser B, Eberl M. Human Vү9/V $\delta 2$ T cells: innate adaptors of the immune system. Cell Immunol 2015;296:10-21.

20. Papatriantafyllou M. T cells: the plastic virtues of a CD4+ T cell. Nat Rev Immunol 2013;13:151.

21. Lees JR, Farber DL. Generation, persistence and plasticity of CD4 T-cell memories. Immunology 2010;130:463-70.

22. Baraldi E, Filippone M. Chronic lung disease after premature birth. N Engl J Med 2007;357:1946-55.

23. Bonadies L, Zaramella P, Porzionato A, Perilongo G, Muraca M, Baraldi E. Present and future of bronchopulmonary dysplasia. J Clin Med 2020;9:1539.
24. Álvarez-Fuente M, Moreno L, Mitchell JA, Reiss IK, Lopez P, Elorza $D$, et al. Preventing bronchopulmonary dysplasia: new tools for an old challenge. Pediatr Res 2019;85:432-41.

25. Zaramella P, Munari F, Stocchero M, Molon B, Nardo D, Priante E, et al. Innate immunity ascertained from blood and tracheal aspirates of preterm newborn provides new clues for assessing bronchopulmonary dysplasia. PloS One 2019;14:e0221206.

26. Biasucci G, Rubini M, Riboni S, Morelli L, Bessi E, Retetangos C. Mode of delivery affects the bacterial community in the newborn gut. Early Hum Dev 2010;86(1 Suppl):13-5.

27. Grönlund MM, Lehtonen OP, Eerola E, Kero P. Fecal microflora in healthy infants born by different methods of delivery: permanent changes in intestinal flora after cesarean delivery. J Pediatr Gastroenterol Nutr 1999;28:19-25.

28. Shen CM, Lin SC, Niu DM, Kou YR. Labour increases the surface expression of two toll-like receptors in the cord blood monocytes of healthy term newborns. Int J Paediatr 2009;98: 959-62.

29. Perez-Sepulveda A, Torres MJ, Khoury M, Illanes SE. Innate immune system and preeclampsia. Front Immunol 2014;5:244.

30. Bonney EA. Preeclampsia: a view through the danger model. J Reprod Immunol 2007;76:68-74. 